\title{
Black hole masses in type II AGNs from the Sloan Digital Sky Survey
}

\author{
W. Bian, ${ }^{1,2}$ Q. $\mathbf{G u}^{3}$ and Y. Zhao ${ }^{3}$ \\ ${ }^{1}$ Dept. of Physics and Inst. of Theor. Phys., Nanjing Normal Univ., Nanjing 210097, China \\ ${ }^{2}$ Key Laboratory for Particle Astrophysics, Institute of High Energy Physics, Chinese \\ Academy of Sciences, Beijing 100039, China \\ ${ }^{3}$ Department of Astronomy, Nanjing University, Nanjing 210093, China \\ email: bianwh@ihep.ac.cn
}

\begin{abstract}
The stellar population synthesis method is used to model the stellar contribution for a sample of 209 type II AGNs at $0.3<z<0.83$ from the Sloan Digital Sky Survey. The reliable stellar velocity dispersions are obtained for 33 type II AGNs with significant stellar absorption features. We use the formula of Greene \& Ho to obtain the corrected stellar velocity dispersions $\left(\sigma_{*}^{c}\right) .20$ of which can be classified as type II quasars. The SMBHs masses and the Eddington ratios are calculated. We measure the gas velocity dispersion $\left(\sigma_{g}\right)$ from NLRs, and find that the relation between $\sigma_{g}$ and $\sigma_{*}^{c}$ becomes much weaker at higher redshifts than at smaller redshifts. We find that the deviation of $\sigma_{g}$ from $\sigma_{*}^{c}$ is correlated with the Eddington ratio.
\end{abstract}

Keywords. Galaxies: active - galaxies: nuclei - quasars: emission lines

\section{Stellar bulge velocity dispersion in type II AGNs}

According to the unification model, AGNs are classified into two classes depending on whether the central engine and broad-line regions (BLRs) are viewed directly (type I AGNs) or are obscured by medium (type II AGNs). Type II quasars are the luminous analogs of low-luminosity type II AGNs. Some methods have been used to discover type II quasars, but only a handful have been found. The sparsity of type II quasars is possibly due to the torus evolution with the luminosity. Here we used the sample of type II Quasars at redshifts $0.3<z<0.83$ from the Sloan Digital Sky Survey (SDSS) (Zakamsa et al. 2003). The fibers in the SDSS survey have a diameter of 3", and contains the host galaxy light. It is the key point to accurately measure the stellar velocity dispersion $\left(\sigma_{*}\right)$.

We first modelled the stellar contribution in the SDSS spectra through the stellar population synthesis code, STARLIGHT (version 2.0, Cid Fernandes et al. 2001). The code does a search for the linear combination of Simple Stellar Populations (SSP) to match a given observed spectrum $O_{\lambda}$. We focus on the strongest stellar absorption features of Ca II $\mathrm{K}$ and the G-band. We find that the fitting goodness (chi-square value) depends not only on the $\mathrm{S} / \mathrm{N}(>5)$, but also on the absorption lines equivalent widths (EW of Ca II $\mathrm{K}$ line $>1.5 \AA$ ). At last we select 33 type II AGNs, which had shown significant stellar absorption features and are well fitted to derive reliable measurements of stellar velocity dispersion. For more details, please refer to Bian et al. (2006).

\section{The extinction correction of the $[\mathrm{O}$ III] $\lambda 5007$ luminosity}

We used the $[\mathrm{O}$ III $] \lambda 5007$ line as a tracer of AGNs activity. However, the $[\mathrm{O}$ III $] \lambda 5007$ luminosity is subject to extinction by interstellar dust in the host galaxy and in our Galaxy. The extinction correction is usually corrected by using the Balmer decrement, which is regarded as the best approximation. For 18 out of these 33 objects with available 
$\mathrm{H} \alpha$ measurements in the stellar-light subtracted spectra, we used the relation $L_{[\mathrm{OIII}]}^{\mathrm{c}}=$ $L_{[\mathrm{OIII}]}\left[\frac{\left(\mathrm{H}_{\alpha} / \mathrm{H}_{\beta}\right)_{\text {obs }}}{\left(\mathrm{H}_{\alpha} / \mathrm{H}_{\beta}\right)_{0}}\right]^{2.94}$ to obtain the extinction correction of the [O III $] \lambda 5007$ luminosity. If we used the $L_{[\mathrm{OIII}]}$ criterion of $3 \times 10^{8} \mathrm{~L}_{\odot}$, which corresponds to the intrinsic absolute magnitude $M_{B}<-23$ (Zakamsa et al. 2003), 14 out of these 18 objects can then be classified as type II quasars. Using the SDSS AGN catalogue at MPA/JHU (Kauffmann et al 2003), we obtained the extinction factor at $5007 \AA, \log \left(\frac{L_{[O I I I]}^{c}}{L_{[O I I I}^{c}}\right)$. We tried to obtain a relation between $\frac{L_{[O I I]}^{c}}{L_{[O I I !}}$ and other observational parameters, such as $\sigma_{*}, L_{[O I I I]}, L_{H \beta}$. However, no correlation is found. More careful work is required to deal with this problem in the future.

\section{The SMBHs masses and Eddington ratios in type II AGNs}

There are some methods to calculate the SMBHs masses in AGNs. With the broad emission lines from BLRs (e.g. $\mathrm{H} \beta, \mathrm{Mg}$ II, CIV; $\mathrm{H} \alpha$ ), the reverberation mapping method and the empirical size-luminosity relation can be used to derive the virial SMBHs masses in AGNs. The obscuration of BLRs makes above method infeasible to derive SMBHs masses. However, we can use the well-known $M_{\mathrm{BH}}-\sigma_{*}$ relation to derive SMBHs masses if we can accurately measure the stellar bulge velocity dispersion $\left(\sigma_{*}\right)$.

Recently, Greene \& Ho (2006) used the direct-fitting method to study the the systematic biases of $\sigma_{*}$ from different regions around $\mathrm{Ca}$ II triplet, $\mathrm{Mg}$ Ib triplet, and $\mathrm{CaII}$ $\mathrm{H}+\mathrm{K}$ stellar absorption features. Here we used the following formula to obtain the corrected velocity dispersion $\sigma_{*}^{c}$ (Greene \& Ho 2006), $\sigma_{*}^{c}=(1.40 \pm 0.04) \sigma_{*}-(71 \pm 5)$. From this corrected velocity dispersion, we calculated the SMBHs masses. We used the extinction-corrected [O III] luminosity to calculate the bolometric luminosity $L_{\mathrm{bol}}$, $\mathrm{L}_{\mathrm{bol}}=3500 \mathrm{~L}_{[\mathrm{OIII}]}^{\mathrm{c}}$. At last we calculated the Eddington ratio, $L_{\mathrm{bol}} / L_{\mathrm{Edd}}$, where $\mathrm{L}_{\mathrm{Edd}}=$ $1.26 \times 10^{38} \mathrm{M}_{\mathrm{BH}} / \mathrm{M}_{\odot} \operatorname{ergs~s}^{-1}$.

\section{The $\sigma_{\mathrm{g}}-\sigma_{*}$ relation}

The good correlation between stellar velocity dispersion $\left(\sigma_{*}\right)$ and ionized gas velocity dispersion $\left(\sigma_{\mathrm{g}}\right)$ suggests that the gaseous kinematics of NLRs in Seyfert galaxies be primarily dominated by the bulge gravitational potential.

Following Greene \& Ho (2005), we study the $\sigma_{\mathrm{g}}-\sigma_{*}^{c}$ relation for 33 Type II AGNs at redshifts $0.3<\mathrm{z}<0.83$ after measuring the gas velocity dispersion $\left(\sigma_{\mathrm{g}}\right)$ from the narrow emission lines from NLRs, and the stellar velocity dispersion $\left(\sigma_{*}^{c}\right)$ from the CaII $\mathrm{H}+\mathrm{K}$, G-band absorption feature. In Fig. 6, we showed the relation between $\sigma_{g}$ and $\sigma_{*}^{c}$. The relation between $\sigma_{g}$ and $\sigma_{*}^{c}$ becomes much weaker at higher redshifts.

This work has been supported by the NSFC (Nos. 10403005, 10473005, 10325313, 10233030 and 10521001) and the Science-Technology Key Foundation from Education Department of China (No. 206053).

\section{References}

Bian, W., Gu, Q., Zhao, Y., Chao, L. \& Cui, Q. 2006, MNRAS, 372, 876

Cid Fernandes, R., Sodre, L., Schmitt, H. R. \& Leao, J. R. S. 2001, MNRAS 325, 60

Greene, J. E., Ho, L. \& C. 2005, ApJ 627, 721

Greene, J. E. \& Ho, L. C. 2006, ApJ 641, 117

Kauffmann, G. et al. 2003, MNRAS 346, 1055

Zakamsa, N. L. et al. 2003, AJ 126, 2125 